\title{
LA FUNDAMENTAL HOMOGENEIDAD DE LAS TEORÍAS MONETARIAS DE GEORG SIMMEL Y LUDWIG VON MISES
}

\author{
OSCAR VARA CRESPO*
}

Resumen. Este artículo analiza los puntos de confluencia entre las teoría del dinero de Ludwig von Mises y Georg Simmel, tanto en su enfoque metodológico, como en el carácter kantiano del análisis y en sus principales conclusiones sobre el papel del dinero como institución social.

Abstract. This article examines the points of convergente between the theories of money propounded by Ludwig von Mises and Georg Simmel. Consideration is given to agreement in the methodological approach, in the Kantian nature of the analyses, and in the main conclusions regarding the role of money as a social institution.

Palabras clave: filosofía del dinero, teoría del crédito, proceso de mercado, acción humana.

Códigos JEL: B13, B19, B53

* Universidad Autónoma de Madrid. 


\section{Introducción}

Con este ensayo pretendemos confrontar los métodos contenidos tanto en la obra de Georg Simmel La Filosofía del Dinero como en el pensamiento económico de Ludwig von Mises, principal exponente de la Escuela Austriaca de Economía. ${ }^{1}$ De esta confrontación, y aplicando a las dos teorías un método sistemático de análisis, buscamos demostrar que hay una homogeneidad esencial entre ambas aportaciones teóricas. ${ }^{2}$ En concreto, intentaremos demostrar la siguiente tesis: que el método científico aplicado a la cuestión del dinero tanto por el citado Simmel como por Ludwig von Mises es el mismo. ${ }^{3}$

Este método sistemático consistirá en dejar al descubierto, lo más claramente posible, aquellos elementos configuradores de cada una de las teorías de estos dos autores. Es decir, pretendemos mostrar cómo las premisas de dichas teorías son en esencia las mismas.

Pensamos que es especialmente interesante resaltar cómo ya en Simmel están los procedimientos científicos que, con la obra de Mises, dan cuerpo definitivo a la metodología de la Escuela Austriaca de Economía. Especialmente teniendo en cuenta las filiaciones a las que se ha adscrito a Simmel. ${ }^{4}$

\footnotetext{
${ }^{1}$ Mientras que la obra monetaria de Mises se puede encontrar en diversos trabajos, en el caso de Simmel nos centraremos en la parte analítica de su Filosofía del Dinero, que pensamos es la parte más esencial de su trabajo. En esto coincidimos con la valoración que hizo, casi un siglo atrás, Altmann. Ver Altmann, 1903, p. 47.

${ }^{2}$ La relación que deseamos exponer es más concreta que la existente entre la Escuela Austriaca de Economía y la obra de Simmel, relación ésta última ya señalada por otros autores. Sobre esta cuestión, ver, por ejemplo, Laidler D. y Rowe, N. (1980), o Monceri, F. (1998).

${ }^{3}$ Nuestro trabajo también pretende responder a las calificaciones vertidas sobre el libro de Simmel que afirman que no es una obra de teoría económica (ver, p. e., Durkheim, 1900-01) incluida la del propio Simmel (ver prefacio de la Filosofía del Dinero). Ya que, si tenemos éxito en lo que queremos demostrar, esa crítica también sería extensible a la obra de los autores austriacos, tesis que no compartimos.

${ }^{4}$ Sobre esta cuestión, ver Simmel (1990), en concreto, el prólogo escrito por David Frisby.
} 
Ahora bien, de esta homogeneidad sólo nos interesará, en el presente ensayo, un aspecto: el que resalta la importancia fundamental que tienen las concepciones antropológicas en la formación de las teorías económicas. No estamos tentados de intentar una minusvaloración de las aportaciones de Mises a la ciencia económica que exceden enormemente a las de Simmel (es fácil comprobar que La Filosofía del Dinero no es un tratado de teoría económica), ni tampoco sugerir que Simmel fue el creador de un método que se ha atribuido generalmente a Mises. De hecho, ya ha sido analizada la influencia que la obra de Carl Menger, fundador de la Escuela Austriaca, tuvo sobre la teoría monetaria expuesta por Georg Simmel, ${ }^{5}$ así como la valoración negativa del trabajo de Simmel que hizo el propio Menger. ${ }^{6}$

Más bien, queremos resaltar que Simmel y Mises participan de una misma tradición intelectual, aunque puede que no sean, precisamente, desarrollos paralelos. ${ }^{7}$

\section{La concepción antropológica de Georg Simmel y Ludwig von Mises: sus teorías del conocimiento}

Un elemento principal de las teorías económicas suele ser la definición que hacen del agente económico, sencillamente porque es el protagonista y responsable de los fenómenos económicos. En el caso de los dos autores que nos ocupan, esta importancia es mayor, porque la definición del agente económico

5 Ver, especialmente, Monceri (1998) y Laidler y Rowe (1980), p. 97.

${ }^{6}$ Ver Frisby, 1992, pp. 84-5. También señala este autor que Simmel expuso su teoría psicológica del dinero en la Sociedad de Economistas Austriacos en octubre de 1896, donde, presumiblemente, podrían haber estado como oyentes Carl Menger, Friedrich von Wieser o Eugen von Böhm-Bawerk.

7 Laidler y Rowe citan a Fritz Machlup, quien aseguraba que muchos economistas austriacos veían en Simmel un desarrollo paralelo de ideas similares, pero no una fuente de nuevas ideas. Ver Laidler y Rowe, 1980, nota 5, p. 100. Sin embargo, no estamos en condiciones actualmente de iniciar esta investigación. 
configura todo su discurso teórico-económico, tal y como esperamos demostrar.

En concreto, la descripción del agente económico que realizan tanto Georg Simmel como Ludwig von Mises pone el acento, especialmente, en las capacidades cognitivas de la persona. En concreto, ambos entienden la persona desde unas coordenadas filosóficas kantianas. Es más, para describir cómo conocen las personas, y los agentes económicos, utilizan la teoría del conocimiento que enunció Inmanuel Kant en su Crítica de la Razón Pura y afirman que para que el conocimiento humano sea posible es necesario que existan ciertas categorías a priori en la mente humana. ${ }^{8}$ Es decir, es necesario que existan ciertas capacidades en la mente, previas a la ocurrencia de experiencias sensibles, que extraigan y condicionen el conocimiento a partir de los datos de la experiencia sensible. Sin embargo, ambos reconocen que no es posible una enumeración exacta de ellas. ${ }^{9}$ Pero ninguno de los dos se embarca en la enumeración de ellas y admiten las dificultades reales que hay para ello.

Del mismo modo, reconocen que la acción humana, para producirse, también ha de ser gobernada por categorías a priori. Pero, en este caso, sí admiten la posibilidad de enunciarlas. En concreto, definen dos principios ineludibles para la acción humana: el de teleología y el de causalidad.

Mises justifica estas categorías a partir de su definición de la acción humana como toda conducta deliberada y consciente que busca alcanzar ciertos fines con ciertos medios. ${ }^{10}$ Es decir, la acción, por ser consciente y deliberada, está proyectada a la consecución de un fin concreto y es forzosamente teleológica. ${ }^{11}$ Por otro lado, la acción requiere un conocimiento previo concerniente a las circunstancias propias del fin perseguido y de las

\footnotetext{
8 Ver Simmel, 1990, p. 105, y Mises, 1962, pp. 12-4.

9 Ver Simmel, 1990, pp. 113-114 y Mises, 1962, p. 20.

10 Ver Mises, 1996, p. 11.

11 Ver Mises, 1962, p. 36 y 1996, p. 28.
} 
conexiones causales existentes entre la acción a realizar y el fin perseguido que, según Mises, tendrá un fuerte componente experiencial.

The first and basic achievement of thinking is the awareness of constant relations among the external phenomena that affect our sense. A bundle of events that are regularly related in a definite way to others events is called a specific thing and as such distinguished from other specific things. The starting point of experimental knowledge is the cognition that an $A$ is uniformly followed by a $B$. The utilization of this knowledge either of the production of $B$ or for the avoidance of the emergence of $B$ is called action. The primary objective of action is either to bring about $B$ or to prevent its happening.

Whatever philosophers may say about causality, the fact remains that no action could be performed by men not guided by it.

(Mises, 1962, p. 20).

Estas reflexiones de Mises tienen, una por una, antecedentes en la obra de Simmel, quien, por su parte, aborda la cuestión de la acción humana un poco más indirectamente a partir de una polémica: aquella que divide en dos corrientes, la causal y la teleológica, el campo del pensamiento acerca de la acción humana.

If, for instance, an aimless inner unrest drives us to furious activity, then this belongs to the category of instinctual behavior; if we undertake the same activity in order to attain some precise kind of well-being, then it belongs to the category of purposive behavior. (Simmel, 1990, p.204).

Este pensamiento busca alcanzar una meta, y es, por tanto, teleológico. Ahora bien, para la consecución de un fin concreto a veces es necesario embarcarse en cursos de acción indirectos, que exigen diversas acciones intermedias. A estos cursos indirectos los llama Simmel cadenas teleológicas, por estar lanzadas a un final ya pensado. Sin embargo, estos cursos de acción no serían posibles si no hubiera un conocimiento, o creencia de 
conocimiento, sobre las vinculaciones causales entre la acción y la consecución del fin.

«a teleological chain can never occur unless the causal connections between its elements are known. The purpose repays this service by providing the psychological impulse to seek out causal relations. Thus, the factual and logical possibility of the teleological chain depends upon the causal relation, but the interest of this causal chain, its psyhological possibility, arises from the pursuit of an end. (Simmel, 1990, p. 208).

Esta visión del conocimiento precondiciona su veracidad, o mejor dicho, el estatuto de esta veracidad. Cuestión que nos interesa especialmente para entender el propio carácter que Simmel y Mises conceden a las instituciones sociales como conocimiento adquirido no sólo por los individuos, sino por las colectividades.

\section{Verdad y conocimiento en Simmel y Mises}

\subsection{La teoría de la verdad de Georg Simmel}

Como ya hemos visto, la acción se basa en el conocimiento y éste en ciertas categorías a priori. Pero, ¿qué carácter tiene este conocimiento? ¿Qué tipo de información nos proporciona sobre la realidad circundante? ¿Qué representa como guía de las acciones humanas? ¿Qué verdad sobre la realidad nos da el conocimiento entendido a la kantiana? Veamos, en primer lugar, lo que Simmel tiene que decir de esas cuestiones.

Simmel ataca las teorías de las verdad que nacen de las posiciones filosóficas dogmáticas, escépticas y críticas, pues todas perecen ante la prueba de lo que denomina "problema del fundamento del principio»: es decir, el problema que plantea el fundamento sobre el que se enuncia cualquier verdad. Ni los dogmáticos pueden escapar a que la verdad a la que se aferran ha de estar sustentada en otra, ni los escépticos a la propia contradicción de afirmar que no hay ninguna verdad, ni los críti- 
cos a la imposibilidad de demostrar si la experiencia sobre la que fundan su conocimiento es válida como principio último. Por ello Simmel adopta una solución relativista. Puesto que toda verdad ha de recibir su veracidad de otra, sólo hay dos alternativas: o bien, un regreso infinito de verdad en verdad que nada enseña; o bien, lo que Simmel denomina la reciprocidad probatoria entre proposiciones o, más brevemente, el relativismo. ${ }^{12}$

Simmel nos explica que la referencia a la prueba recíproca no es válida cuando se refiere a deducciones particulares, pues es mero círculo vicioso. Sin embargo, cuando alude a la totalidad de nuestro conocimiento queda forzosamente apresado en esta forma. La totalidad del conocimiento para el hombre tiene validez en algo que está fuera de esa totalidad. Es decir, el conocimiento es válido y cierto en relación con nosotros, a nuestras necesidades de supervivencia y a los resultados que esperamos de nuestras acciones.

Initially, truth is not useful because it is true, but vice-versa. We dignify with the name of 'truth' those representations that, active within us as real forces or motions, incite us to useful behaviour. Thus there are as many basically different truths as there are different organizations and conditions of life. (Simmel, 1990, p. 107).

La totalidad de estos conocimientos sólo obtienen su validez en relación con la organización psico-física a la que se refieren, a sus condiciones de vida y a la necesidad de su actividad.

A ese conocimiento poseído por un individuo, es decir, el contenido de lo que conoce de modo consciente, lo denomina Simmel espíritu subjetivo. La verdad a la que se atiene el sujeto estará formada por estas representaciones de la realidad y serán sometidas a constante prueba y reformulación, por lo que las representaciones que van quedando no serán otras que las «favorables a la existencia». Será desde ellas como la persona

\footnotetext{
12 Simmel, 1990, p. 106 y 115.
} 
pensará el mundo circundante y las novedades que se le presenten. ${ }^{13}$

Por lo tanto, Simmel está afirmando que el conocimiento tiene el carácter de herramienta para la persona en tanto orienta su acción y cuya verdad depende de su utilidad, no de la verdad que predican sobre la realidad. ${ }^{14}$

\subsection{La teoría de la verdad de Ludwig von Mises}

En lo referente a estas mismas cuestiones, Mises inicia su investigación suponiendo que la estructura lógica de la mente humana está dada. Esto es, que la dotación de categorías a priori sobre las que se construye el conocimiento humano, puede tomarse como dada.

Esta afirmación posee implicaciones especialmente fuertes para la teoría de la verdad. En primer lugar, este supuesto implica la aceptación de una posición filosófica idealista; en segundo lugar, este idealismo implica que el conocimiento en cada persona es un conjunto de proposiciones de carácter tautológico o silogístico que se va construyendo sobre la estructura lógica de la mente y por medio de la argumentación y la experiencia; $\mathrm{y}$, en tercer lugar, implica que de estas proposiciones no se puede asegurar más que su coherencia lógica, no su veracidad.

Entonces, ¿qué conocimientos son verdaderos? ¿Bajo qué supuestos se sostiene un conocimiento como verdadero? Mises sigue un doble camino, a diferencia de Simmel, en la consideración de la verdad del conocimiento, pues distingue entre lo que de verdad tiene el conocimiento de la naturaleza y lo que de verdad tiene el del hombre: por un lado, entiende que la verdad del conocimiento sobre la naturaleza depende de su rela-

${ }^{13}$ Simmel, 1990, p. 107.

${ }^{14}$ Sobre el carácter instrumentalista del pensamiento de Simmel, ver Laidler y Rowe, 1980, p. 98, en donde se le relaciona en este punto con Brunner y Meckling y, sobre todo, con Milton Friedman. 
ción con el hombre. Es decir, Mises afirma que, respecto de la naturaleza, el hombre adquiere un conocimiento del que sólo puede decir si le es útil o no, nunca si es verdadero. Se debe esta imposibilidad a que extraemos los fundamentos sobre los que se basan nuestros razonamientos deductivos sobre la naturaleza de la experiencia y de la inducción, y éstos son una guía siempre insuficiente para conformar teoremas universales. ${ }^{15}$ En la misma línea, entiende Mises que las hipótesis son siempre elaboraciones intelectuales de la experiencia y que, por ello, no se reciben inmediatamente de la experiencia sino que están cargadas ellas mismas de teoría. Mises concluye de todo ello que hay un único criterio de validez aplicable a las ciencias naturales que será instrumentalista: será verdad aquel conocimiento que permita alcanzar los objetivos que el hombre persigue, aun cuando no constituya la verdad sobre la realidad a la que se refiere. ${ }^{16}$

Respecto del conocimiento sobre el propio hombre, al nacer de él mismo y ser para sí un objeto de conocimiento inmediato, sí se puede predicar verdad. Mises apoya este postulado en dos afirmaciones: en primer lugar, la acción humana es cogenérica con el pensamiento humano y, por tanto, está sujeta a categorías a priori que, a su vez, son conocibles porque la estructura lógica de la mente es única, está dada y el hombre accede a ella inmediatamente; en segundo lugar, todas las ciencias sociales se desgajan de una ciencia más general, que es la ciencia de la acción humana.

La diferencia esencial entre el fundamento del principio de un argumento sobre la naturaleza y otro sobre el hombre radica en la aprioricidad de las hipótesis que fundan el segundo respecto del primero. Esta aprioricidad quiere decir que, por instrospección, el hombre accede a un conocimiento cierto acerca de su mente, de las posibilidades de su conocimiento y, sobre todo,

\footnotetext{
15 Ver Mises, 1981, p. 9.

16 Ver Mises, 1962, pp. 36, 43 y 62; y Mises, 1996, p. 23-4.
} 
de la acción humana, construyendo teoremas praxeológicos verdaderos. Su verificación, por tanto, no es empírica, sino lógica. ${ }^{17}$

Every theorem of praxeology is deduced by logical reasoning from the category of action. It partakes of the apodictic certainty provided by logical reasoning that starts from an a priori category (Mises, 1962, p. 44).

\section{Las instituciones sociales}

Las aclaraciones previas, como veremos, adquieren en este apartado su pleno significado. Comencemos diciendo que los dos autores ven en las instituciones sociales formas de hacer las cosas, de intentar alcanzar ciertos fines. Por tanto, entienden que son un tipo de conocimiento sobre el mundo y cuya veracidad será instrumental. Veamos en concreto cómo desarrolla cada uno su intelección del problema.

Simmel inicia su investigación sobre las instituciones sociales afirmando que cada persona porta consigo un conocimiento consciente que le es peculiar, su espíritu subjetivo, que, sin embargo, puede hacerse público y comunicarse, discutirse, aceptarse o rechazarse. De este modo, las personas pueden crear un ámbito conjunto de saberes al que Simmel llamará espíritu objetivo (concepto que define siguiendo la tradición idealista de la filosofía de Hegel y Dilthey). ${ }^{18}$ La importancia de este espíritu objetivo para la civilización es enorme:

The objectification of the mind provides the form that makes the conservation and accumulation of mental labour possible; it is the most significant and most far-reaching of the historical categories of mankind. For it transforms into a historical fact what is biologically so doubtful: hereditary transmission. If to be not only a descendant but also an heir denotes the superiority of man

17 Mises, 1962, pp. 18, 71 y 72.

18 Ver Hegel, G.W.F. (1999) y Dilthey, W. (1944). 
over animals, then the objectification of the mind in words and works, organizations and traditions is the basis for this distinction by which man takes possession of his world, or even of any world at all (Simmel, 1990, p. 453).

En resumen, podríamos decir que, según Simmel, cada hombre crea representaciones de la realidad reinventando en su cabeza el mundo que experimenta y sometiendo sus hipótesis a pruebas constantes que el mundo, a su vez, se encarga de refutar o fortalecer. De tal manera que aquellos conocimientos que se comprueban útiles para la consecución de un fin concreto pasan a ser parte del acervo de saberes que cada hombre posee, a constituir el reino de lo teórico. Pero este reino de lo teórico puede ser explicitado en obras literarias, artísticas, religiosas, edificaciones, etc., y ser conocido por otros y transformado por otros. Es decir, puede adquirir autonomía respecto de quien lo produce y desarrollarse posteriormente según su propia lógica, imprevisiblemente.

En cualquier caso, el espíritu así objetivado se transformará en una fuerza que ayudará al sujeto, pero que también le ofrecerá resistencia. En el proceso habrá una reciprocidad mutua en la que el sujeto será influido por el conjunto de conocimientos objetivos y, en dirección opuesta, el sujeto influirá y transformará los contenidos de ese conjunto. Este Espíritu Objetivo será, en cierto modo, la vía de relación entre los objetos y el sujeto, entre el mundo externo real y el individuo, pues en él estará la visión, la representación que el hombre se hace del mundo. Su función no será pasiva. Intervendrá sobre aquel que lo piensa y será afectado por el propio pensamiento. Pero es más, el espíritu objetivo será la representación objetiva de la que el hombre es capaz sobre el mundo, aunque no llegue a descubrir nunca cuál sea su verdadera forma.

Precisamente, de este proceso, y como subconjunto del espíritu objetivo, aparecerán las instituciones sociales que no serán más que aquellas pautas de comportamiento que se hayan com- 
probado, a lo largo del tiempo, más efectivas para la solución en sociedad de ciertos problemas objetivos de la persona. Es decir, una institución social será aquella herramienta que utilizará el individuo, en y con la sociedad, cuando no pueda alcanzar ciertos objetivos con su capacidad personal. ${ }^{19}$

Por su parte, Mises llegará a similares conclusiones al analizar la cooperación social. Según él, la limitación del conocimiento humano impone una condición obligatoria, la incertidumbre, por la que cualquiera se ve empujado a la cooperación con otros seres humanos. El individuo busca siempre en su experiencia pasada aquellas relaciones causales que le muestren las vías de acción más beneficiosas, tanto para la comprensión de las circunstancias del medio natural, como del comportamiento de otros hombres. ${ }^{20}$ Sin embargo, esa comprensión será siempre insuficiente, y por eso aparecerá la incertidumbre ${ }^{21}$ y el reconocimiento de que el trabajo en sociedad y las instituciones sociales son el mejor modo de evitarla:

Society comes into being and develops not by virtue of a moral law imposed on mankind by mysterious powers bent on forcing the individual, against his interest, into subordination to the social whole, but through the action of individuals cooperating in the attainment of ends that they severally aim at, in order to take advantage of the higher productivity brought about by the division of labor (Mises, 1962, p. 42).

Como tales, las instituciones sociales serían, también para Mises, aquellas soluciones históricas concretas a que da lugar la cooperación humana para la consecución de fines concretos. La emergencia de dichas instituciones sociales dependería, primero, de la perspicacia de algunos individuos que acaban concluyendo que unas pautas de comportamiento cooperativas con-

\footnotetext{
19 Ver Simmel, 1990, p. 209.

20 Ver Mises, 1962, p. 49.

21 Ver Mises, 1962, p. 46.
} 
cretas son más favorables que otras y, segundo, de su imitación por parte del resto de la sociedad.

Así, instituciones sociales como el mercado o el dinero no son más que formas de tratar de alcanzar fines que se crean y se mantienen porque, como es patente en el proceso de mercado, «the adjustment of the individual actions of the various members of the market society to the requirements of mutual cooperation», 22 facilita la satisfacción de los fines personales.

Ahora bien, el elemento personal de la acción, el que ésta sea radicalmente individual, impide la solución única, la pauta única, la institución social única para la solución de un mismo problema. En el caso del dinero, como veremos, se traduce en una materialización distinta de la forma monetaria en distintas civilizaciones o en distintos momentos de la historia de una misma civilización.

\section{El dinero}

\subsection{La teoría monetaria de Simmel}

Haremos a continuación un breve resumen de los aspectos más resaltables de la teoría monetaria de Simmel. En primer lugar, atendamos a su tratamiento del origen del dinero:

One assumes that objects that were frequently exchanged and circulated because of their general desirability, and the value of which in relation to other objects was therefore frequently measured, were psychologically most suited to become general standards of value (Simmel, 1990, p. 142).

De lo que se deduciría que el surgimiento del dinero es un proceso espontáneo y no dirigido. ${ }^{23}$ Simmel ilustra ampliamente esta cuestión con numerosos ejemplos. ${ }^{24}$

\footnotetext{
22 Ver Mises, 1996, p. 258.

23 Ver Laidler y Rowe, 1980, p. 98.

24 Ver Simmel, 1990, pp. 143-151.
} 
En segundo lugar, y en lo referente a la naturaleza del dinero, Simmel, en línea con su teoría del conocimiento y de la verdad, afirma que es el prototipo de institución social entendida como instrumento para la acción..$^{25}$ Es la herramienta perfecta, ya que le permite alcanzar aquellos objetivos que están fuera de su alcance por sus esfuerzos personales.

Money is the purest reification of means, a concrete instrument which is absolutely identical with its abstract concept; it is a pure instrument (Simmel, 1990, p. 211).

Respecto de las necesidades que satisface, a los objetivos que ayuda a cumplir, Simmel separa dos planos distintos del dinero: por un lado, tenemos su función, la de ser relación valorativa recíproca (lo que lleva a la pregunta sobre cómo podría ser perfecta esa relación); y, por el otro, la substancia sobre la que se materializa, es decir, el hecho de que el dinero ha sido históricamente un valor como lo son también los otros bienes.

En cuanto a la función, el dinero ayuda a enfrentar las valoraciones subjetivas de los individuos permitiendo el intercambio. Sin embargo, sería deseable que esa operación fuera perfecta, que el dinero no influyera en las valoraciones individuales ni las alterara. Así, los cambios de los precios de las cosas responderían a cambios en las relaciones entre esas cosas y no entre las cosas y el dinero. De esta manera el valor del dinero sería verdaderamente estable.

Pero esta cualidad no la cumplirá el dinero sino en cuanto pierda el segundo plano al que nos referíamos, es decir, su substancia, su carácter de valor concreto. El dinero ha de ser mero signo, un símbolo sin valor substancial (sin el sustento de valor que le proporcionaría la substancia sobre la que se encontrara materializado el dinero), un signo que sólo posea un valor funcional ${ }^{26}$

\footnotetext{
25 Ver Laidler y Rowe, 1980, p. 101.

${ }^{26}$ La distinción entre el valor substancial y el funcional es muy importante aquí. El primero podemos definirlo como aquel que proviene del medio en el que se ma-
} 
derivado de su capacidad de intervenir en la relación establecida entre las cosas deseadas, por medio de la cual aquéllas se convierten en valores económicos. Simmel está convencido de que lo propio del dinero es tender a espiritualizarse y a ser sólo símbolo. ${ }^{27}$

Sin embargo, hay que preguntarse si es posible un dinero así. ${ }^{28}$ Simmel nos da un argumento en contra y otro a favor: en primer lugar, resalta que no es posible comparar cantidades de dos objetos que sean cualitativamente diferentes; mientras que, en segundo lugar, afirma que esa comparación se podría hacer si las variaciones de cantidad de los objetos estuvieran vinculadas causalmente, cuando el cambio de una fuera resultado del cambio de la otra, y éstos se produjeran con una proporción conocida. ${ }^{29}$ Sin embargo, acabará reconociendo que ambos argumentos son contrarios a la espiritualización del dinero en la práctica.

Simmel desarrolla el argumento favorable de la siguiente manera: en primer lugar, el nexo causal entre los objetos y el dinero, y que hace que éste sea el valor recíproco de aquellos, es el hombre: «commodities and money may not be measurable by each other in general. It is sufficient that both play a part in human life, within the system of men's practical ends, for the quantitative modification of one to become and index of the other». ${ }^{30}$

No obstante, esto no nos dice de qué manera quedarían vinculadas las variaciones de las cantidades de los objetos que se valoran. Esto es, nos queda saber cómo se fijarían los precios. Es preciso, exige Simmel, aceptar como presupuesto absoluto

terializa el dinero (es decir, de aquello en lo que está representado y que generalmente es una mercancía), mientras que el valor funcional es el que, renunciando a toda valoración proveniente de su substancia, reside exclusivamente en la función monetaria, en la capacidad de servir como medio de cambio.

27 Ver Simmel, 1990, p. 198.

28 Ver Simmel, 1990, p. 131.

29 Ver Simmel, 1990, p. 132-133.

30 Ver Simmel, 1990, p. 137. 
que la suma de todas las mercancías vendibles sea equivalente a la suma de todo el dinero. Si se presupone esto, la igualdad entre el valor de una mercancía y el valor de una cantidad de dinero supone, también, la igualdad de dos quebrados cuyos denominadores serán la suma de todas las mercancías y la suma de todo el dinero, respectivamente, y cuyos numeradores serán las cantidades concretas de mercancía y dinero antes aludidas. La estabilidad del valor del dinero, tal y como ha sido definida arriba, se produciría en esa igualdad de los quebrados, cuando el dinero que se da a cambio de una mercancía representa la misma proporción respecto de la masa general de dinero que la mercancía respecto de la masa general de mercancías. Sólo así el dinero expresaría, en términos prácticos, la relación valorativa recíproca de las cosas en sí misma y cumpliría con su concepto esencial, sin afectar a la relación de las cosas entre sí. El dinero sería fiel a su deber ser, es más, el precio sería el «adecuado».

Mas, en la realidad práctica que estudia la ciencia económica esto no es así. El dinero, cualquiera que haya sido la forma adoptada a lo largo de su historia, siempre se ha materializado en algún valor de uso, esto es, en alguna mercancía. Y, si bien el valor substancial de ese dinero, asociado al valor de uso de la mercancía concreta, ha ido decayendo en favor del valor funcional, «the more extensive and diversified are the services it performs and the more rapidly it circulates» (Simmel, 1990, p. 143), nunca ha podido desembarazarse de él sin un coste elevado como el de la inflación.

El dinero que, como realidad que se materializa en diversas formas a lo largo de la historia, busca adquirir un carácter puramente simbólico, no puede deshacerse de un resto de valores substanciales por un doble motivo. ${ }^{31}$ Primero, porque el individuo es incapaz, por sí mismo, de comprender y tener en cuenta la relación que deben guardar las sumas de dinero y de mercan-

31 Ver Simmel, 1990, p. 158. 
cías concretas a intercambiar con las sumas generales correspondientes de ambas para que el dinero sea un mero símbolo. El segundo motivo reside en la importancia que tiene el dinero como elemento de la circulación, pues el hecho de una circulación creciente que implicara un denominador creciente en la fracción del dinero obligaría a crecimientos continuos de los precios. Si el crecimiento de la circulación está provocado por emisiones de papel moneda realizadas por el gobierno, el resultado de esos aumentos de precios sería, también, un expolio tanto a los vendedores de mercancías como a los compradores.

Simmel opina que sólo los metales nobles o la vinculación del valor del papel moneda al valor de aquellos será capaz de, quizá, evitar esos problemas. ${ }^{32}$ En todo caso, su énfasis se pone en lo demostrativas que estas observaciones son de las relaciones por las cuales el dinero no perderá nunca su valor substancial.

Por otra parte, critica también la teoría, muy generalizada entre los economistas, que afirma que un aumento de la cantidad de dinero que se repartiera equitativamente no produciría efectos sobre los precios ni los intercambios. ${ }^{33} Y$ esto en virtud de las nuevas relaciones que el dinero nuevo crearía con los precios, además de determinado carácter psicológico de los precios que, al permanecer durante algún tiempo, adquieren cierta solidez y rigidez. ${ }^{34}$

\subsection{La teoría monetaria de Mises}

Para Mises, el dinero es una estrategia de intercambio forzada por la ineficiencia del intercambio directo. ${ }^{35}$ Cuando el trueque no permite la satisfacción de las necesidades, porque el agente no encuentra en su entorno social a nadie dispuesto a cambiar

32 Ver Simmel, 1990, p. 160.

${ }^{33}$ Laidler y Rowe ven aquí una crítica al ensayo de David Hume Of Money de quien Simmel habría sacado sus ideas sobre la inflación. Ver Laidler y Rowe, 1980, p. 100.

${ }^{34}$ Simmel, 1990, p. 168.

35 Ver Mises, 1996, pp. 405 y ss. 
las cantidades concretas de algo que tiene por las cantidades concretas de algo que quiere, sucederá una de estas dos cosas: o bien que el agente desista y se dedique por sí mismo a la producción de lo que necesita, o bien que recurra al intercambio de bienes más vendibles con el objetivo de realizar un intercambio indirecto. Este último curso de acción implica una perspicacia por parte del individuo: la que le lleva a entender que hay ciertos bienes cuyo valor de uso subjetivo es mayor en la consideración general de la sociedad. El mayor valor de uso subjetivo aparece como una condición elemental en el origen del dinero.

En resumen, Mises defiende que la existencia de dinero depende, primero, de la capacidad cognoscitiva del agente: el individuo comprende que hay un estado de cosas más favorable que el que vive. Segundo, de la función empresarial: el agente se apercibe de una solución aparente para su orden mental peculiar. Y, tercero, de la adopción de pautas de comportamiento: el uso de un cierto tipo de bien para adquirir, indirectamente, aquello que se desea no pasa inadvertido para otros agentes que, a su vez, adoptan unos usos parecidos.

Debido a esta comprensión histórica del origen del dinero, afirman que es un error valorar el dinero, no por su «valor de uso subjetivo» como cualquier otro bien, sino por su «valor de cambio objetivo», es decir, por las cantidades dadas y concretas de bienes que puede comprar. Mises señala que ningún bien puede ser objeto de cambio respecto de otro, no puede tener un «valor de cambio objetivo», si antes no se han enfrentado dos «valoraciones de uso subjetivas», lo cual implica un razonamiento circular: por un lado, su «valor de uso subjetivo» reside en que ya tiene un «valor de cambio objetivo», pero éste a su vez depende de un «valor de uso subjetivo» que depende a su vez de uno de cambio, y así indefinidamente.

Mises acusa a la economía clásica de evitar la cuestión separando la teoría de la utilidad marginal de la teoría monetaria (en suma, de renunciar a explicar el valor del dinero con las mismas 
herramientas usadas para explicar la formación de los precios de cualquier bien). Una renuncia en la que colabora la visión agregada de la teoría cuantitativa.

Es decir, Mises afirma que las teorías monetarias basadas en la teoría cuantitativa no resuelven la paradoja del valor del dinero y recurre para explicarlo a la teoría del surgimiento y evolución de las instituciones sociales, ya que el dinero es, asimismo, fruto de un proceso evolutivo desarrollado en la historia. La solución la da el que llamó teorema regresivo, ${ }^{36}$ según el cual, el poder adquisitivo presente del dinero depende del que tuvo en el pasado inmediato, lo que retrotrae el valor del dinero de generación en generación hasta el momento en que el dinero era un bien o mercancía del que alguien hizo uso para obtener, indirectamente, un bien que perseguía y no podía adquirir directamente. Es decir, «a historically continuous component is contained in the objective exchange value of money» (Mises, 1980, p. 133.)

Sin embargo, una vez adoptado el bien como medio de cambio, esta misma función monetaria se añade como elemento de valoración para el individuo, existiendo ambos factores en la determinación del valor del dinero: el perteneciente a los usos industriales a los que se pueda aplicar el material del medio de cambio, así como el correspondiente a sus usos como dinero.

En resumen, el dinero existe por una necesidad económica: la de intercambiar. Una necesidad que el trueque o cambio directo no elimina. Ahora bien, el dinero no se inventa, sino que se descubre. La concreta existencia de la condición de dinero en un bien depende de su deseabilidad, de que un número suficiente de personas sientan por el bien una alta valoración subjetiva de uso, de que alguien entienda su potencial beneficio y de que, también, ese bien posea ciertas cualidades técnicas que favorezcan su desempeño como dinero. Es decir, su función de medio de cambio no es previa a su deseabilidad como bien, sino poste-

36 Ver Mises, 1996, p. 409. 
rior. Tal concepción llevó a Mises, y a la escuela austriaca en general, a oponerse a la existencia del dinero fiduciario, denunciando los atropellos que implica para la hacienda de los ciudadanos y para el discurso tranquilo de los negocios. Por supuesto que es consciente de que cuanto más tiempo ocupe ese bien el papel de medio de cambio, más tenderá a ser valorado por esta función concreta y menos por su substancia. Además, la evolución de la materialización del dinero hacia formas cada vez más apropiadas a su función da la impresión de una desligación entre la función y el bien que la porta. Sin embargo, la normalidad del uso del dinero y su evolución no debe, según los austriacos, concluir en una «espiritualización» del dinero como la calificaba Simmel. El medio de cambio no puede perder su soporte de valor de uso subjetivo sin que se provoquen consecuencias indeseadas. Por ello, defienden la existencia de coeficientes de caja del $100 \%$ para la banca comercial. ${ }^{37}$

La argumentación de su oposición a la reserva fraccionaria se inicia con una critica a la teoría cuantitativa del dinero por ser inadecuada, primero, para explicar el valor del dinero y, segundo, para explicar los efectos económicos de los aumentos de la cantidad de dinero en circulación.

Respecto a la primera, Mises critica que la teoría cuantitativa no se basa en una teoría general del valor. Más bien, lo que hace es apoyarse en los movimientos agregados de la oferta y la demanda de dinero para describir los efectos sobre el valor del dinero. ${ }^{38}$ Es decir, se basa en la idea de que hay una conexión entre las variaciones del valor del dinero y las variaciones en las relaciones entre demanda y oferta del dinero. ${ }^{39}$

La clave de esta insuficiencia de la teoría cuantitativa está en su enunciación agregada, porque no es la demanda de la comu-

\footnotetext{
37 Sobre esta cuestión, ver Huerta de Soto (1996).

38 Mises, 1980, pp. 151-53.

39 Mises, 1980, p. 152.
} 
nidad la que determina los cambios sobre los precios, sino las de los individuos. ${ }^{40}$

Por eso, cuando la cantidad de dinero en circulación varía de tal modo que, para un gran número o todos los agentes de la economía, sus respectivos saldos monetarios exceden lo que sus deseos demandan, el efecto sobre el nivel de precios no puede explicarse en la sede agregada, sino en la más elemental, en la individual. Lo cual no niega el enunciado principal de la teoría cuantitativa, pero sí la hipótesis de neutralidad del dinero, porque un aumento de la cantidad de dinero, a partir de una situación de equilibrio, no afecta a cada individuo de la misma manera. ${ }^{41}$

Ni siquiera por la circulación del dinero, al contrario de lo que suele indicar la teoría cuantitativa en su visión global.

Since the increased quantity of money is received in the first place by a limited number of economic agents only and not by all, the increase of prices at first embraces only those goods that are demanded by these persons; further, it affects these goods more than it afterward affects any others. When the increase of prices spreads farther, if the increase in the quantity of money is only a single transient phenomenon, it will not be possible for the differential increase of prices of these goods to be completely maintained; a certain degree of adjustment will take place. But there will not be such a complete adjustment of the increases that all prices increase in the same proportion. The prices of commodities after the rise of prices will not bear the same relation to each other as before its commencement; the decrease in the purchasing power of money will not be uniform with regard to different economic goods (Mises, 1980, pp. 162-3).

Por eso, afirma que no se puede considerar el dinero como neutral ni creer que es posible que sea plenamente neutro. Es más, entiende que el dinero no puede ser neutral si es que ha de cumplir con su función de señalizar los precios. ${ }^{42}$

\footnotetext{
40 Mises, 1980, p. 152-3.

41 Mises, 1980, p. 160-2.

42 Mises, 1996, p. 418.
} 
Para que el dinero fuera neutral, o, lo que es lo mismo, que su valor fuera estable, debería comprar lo mismo de cada bien en cada momento del tiempo, lo que, a su vez, implicaría que los bienes entre sí deberían mantener también sus relaciones. Una posibilidad que, situada en un marco de agentes que valoran la realidad peculiar y subjetivamente, se antoja descabellada.

\section{Conclusiones}

Las obras de Simmel y Mises guardan una similitud que no puede pasarse por alto y que, tras la exposición desarrollada, debe ser expuesta más claramente en forma de conclusiones:

1. ${ }^{a}$ En primer lugar, hemos constatado que ambos autores utilizan un mismo método científico, el individualismo metodológico.

2. ${ }^{a}$ En segundo lugar, los dos caracterizan a los agentes económicos de la misma manera, es decir, a través de una teoría del conocimiento kantiana.

3. ${ }^{a}$ En tercer lugar, de esta caracterización se sigue que el conocimiento que adquieren las personas sobre la realidad que les circunda nunca es verdadero si por tal entendemos la correspondencia de lo pensado y del pensamiento. Es decir, el conocimiento sobre la realidad circundante es meramente instrumental y su veracidad está exclusivamente en función de que consiga satisfacer las necesidades individuales. Además, hemos visto cómo para Mises sí es posible un conocimiento sobre los seres humanos y que parte, a su vez, de la propia teoría del conocimiento kantiana.

4. ${ }^{\text {a }}$ En cuarto lugar, la imposibilidad de conocimientos ciertos sobre la realidad hace que los problemas más complejos que tiene el ser humano deba resolverlos por medio de un conocimiento compartido y creado en la historia entre todos los hombres. Es decir, cada hombre se ve impelido a adoptar 
algunos comportamientos que ve en otros porque observa que son más favorables para la consecución de ciertos fines que le son propios, no por otra razón. Cuando esas pautas de comportamiento se generalizan y se produce su conversión en uso común es como si ese conocimiento hubiera sido elevado de categoría. Así aparece la institución social, esto es, la pauta de comportamiento que se acepta como vía natural de resolución de ciertos problemas.

5. ${ }^{\text {a }}$ Entre estas pautas de comportamiento encontramos el dinero, que sería una institución social que tiene por objetivo la satisfacción de las necesidades individuales dentro de una comunidad o sociedad.

6. ${ }^{a}$ Afirman que el dinero ha de poseer un valor intrínseco de uso para poder realizar adecuadamente su función monetaria. Sin embargo, es en esta cuestión donde se separan más Simmel y Mises. Para el primero, esto es un efecto inevitable que le impide al dinero ser lo que debe, es decir, mero símbolo. Para el segundo, el que el dinero posea un valor de uso es una condición esencial al dinero que le hace cumplir perfectamente con su misión, es decir, con la transmisión de información en los mercados.

7. ${ }^{\text {P }}$ Por último, ambos critican la hipótesis de neutralidad del dinero. 


\section{REFERENCIAS BIBLIOGRÁFICAS}

Altmann, S.P. (1903), «Simmel's Philosophy of Money», The American Journal of Sociology, 9, pp. 46-68.

Dilthey, W. (1944). El Mundo Histórico. México: Fondo de Cultura Económica. Edición original de 1923, 1927 y 1931.

DURKHEIM, E. (1900-01), «Review of Philosophie des Geldes», en Formal Sociology, Larry Ray (ed.), Aldershot: Edward Elgar Publishing Limited, 1991, pp. 61-66. Originalmente en L'Année Sociologique, 5, 1900-01, pp. 140-45.

FrISBY, D. (1992), Georg Simmel and Since: Essays on Georg Simmel's Social Theory. Londres: Routledge.

Hegel, G.W.F. (1999), Enciclopedia de las Ciencias Filosóficas en Compendio. Madrid: Alianza Editorial. Edición original de 1830.

Huerta De Soto, J., Dinero, Crédito Bancario y Ciclos Económicos. Madrid, Unión Editorial.

LAIDLER, D. y ROWE, N. (1980), «Georg Simmel's Philosophy of Money: A Review Article for Economists», Journal of Economic Literature, vol. XVIII, marzo, pp. 97-105.

MiSES, L. VON (1962), The Ultimate Foundation of Economic Science, Princton, Van Nostrand Company, Inc.

MISES, L. VON (1980), The Theory of Money and Credit, Indianapolis, Liberty Classics [ed. esp.: La teoría del dinero y del crédito, Unión Editorial, 1997].

Mises, L. VON (1981), Epistemological Problems of Economics. Nueva York, New York University Press.

Mises, L. VON (1996). Human Action. A Treatise on Economics. San Francisco, Fox \& Wilkes. [Ed. esp.: La acción humana. Tratado de economía, Unión editorial, $6^{\text {a }}$ ed. 2001].

MONCERI, F. (1998), «Il Denaro fra Teoria Economica e Filosofia: note sul Rapporto Menger-Simmel», Diritto e Cultura, año VIII, enero-junio, pp. 81-113.

Simmel, G. (1990), The Philosophy of Money. Londres, Routledge. Edited by David Frisby.

SPYKMAN, N.J. (1925), The Social Theory of Georg Simmel. Chicago, University of Chicago Press. 
Weber, M. (1972). «Georg Simmel as Sociologist», en Formal Sociology, Larry Ray (ed.), Aldershot: Edward Elgar Publishing Limited, 1991, pp. 80-5. Originalmente en Social Research, 1972, 39, pp. 155-62.

Weinstein, D. y Weinstein, M.A. (1989), «Simmel and the Theory of Postmodern Society», en B.S. Turner (ed.) (1989), Theories of Modernity and Postmodernity. Londres, Sage. 